CASE REPORT

\title{
Aldosterone synthase deficiency type I with no documented homozygous mutations in the CYP11B2 gene
}

\author{
Malgorzata Wasniewska, Francesco De Luca, Mariella Valenzise, Fortunato Lombardo and Filippo De Luca \\ Istituto di Clinica Pediatrica, Policlinico Universitario, 98123 Messina, Italy \\ (Correspondence should be addressed to F De Luca; Email: fdeluca@unime.it)
}

\begin{abstract}
This case report concerns a girl born from non-consanguineous parents and hospitalized in another hospital at the age of 14 days because of a severe salt-losing syndrome $(\mathrm{Na}=125, \mathrm{~K}=8.6 \mathrm{mEq} / \mathrm{l})$. In spite of normal genitalia, diagnosis of 21-hydroxylase deficiency was assessed on the basis of a slightly increased 17-OH-progesterone serum level $(6.4 \mathrm{ng} / \mathrm{ml})$. The onset of both hydrocortisone and $9 \alpha$ fluorohydrocortisone therapy was followed by a resolution of the clinical picture. At the age of 60 days she was admitted to our clinic for a re-evaluation of the diagnosis. Steroid hormone serum levels were measured after withdrawal of therapy and diagnosis of corticosterone methyl oxidase (CMO) deficiency type I was definitely established in the light of the biochemical results: i.e. very low 18hydroxycorticosterone (18-OH-B) serum levels $(20 \mathrm{pg} / \mathrm{ml})$, an abnormally high corticosterone/18$\mathrm{OH}-\mathrm{B}$ serum ratio (306.5) and an abnormally low 18-OH-B/aldosterone serum ratio (2.1). This autosomal recessively inherited disorder can be differentiated from CMO type II and other salt-wasting syndromes only on the basis of the serum steroid hormone pattern. After establishing the diagnosis of CMO I deficiency, hydrocortisone therapy was withdrawn whilst treatment with $9 \alpha$-fluorohydrocortisone was begun again, with a satisfactory clinical and metabolic impact. Direct sequences of the patient's DNA were able to identify only a (heterozygous) amino acid substitution in exon 7 of that gene, which is known to have only a small effect on enzyme activity and cannot be the only cause of the patient's phenotype: valine-386-alanine (V386A) GTG $\rightarrow$ GcG. No homozygous mutations in the CYP11B2 gene were observed. This is the first report of a patient with CMO type I who does not carry any homozygous mutation in the entire CYP11B2 alleles, whereas some cases with no mutations in this gene have already been reported in CMO II. The present study seems to be inconsistent with the previously reported correlation of the phenotype and genotype in CMO type I. A reasonable question that might be raised on the basis of our findings in this case report is whether other genes, apart from CYP11B2, are involved in the regulation of terminal aldosterone synthesis.
\end{abstract}

European Journal of Endocrinology 144 59-62

\section{Introduction}

Isolated hypoaldosteronism is a rare cause of salt wasting in infancy and may produce a life-threatening disease, especially in the newborn infant (1). It is an autosomal recessively inherited disorder of terminal aldosterone (Aldo) synthesis leading to selective Aldo deficiency. There are two biochemically different forms which are termed corticosterone methyl oxidase (CMO) or aldosterone synthase deficiency type I and type II and differ in that 18-hydroxycorticosterone (18-OH-B) is deficient in CMO I but overproduced in CMO II, whereas Aldo serum levels are respectively undetectable in CMO I and low to low-normal in CMO II.

Both disorders are characterized by the same clinical picture: frequent vomiting and severe salt loss in the first weeks of life, failure to thrive, and growth retardation. In most of the published clinical case reports, no differences in the severity of clinical signs between infants with CMO I and CMO II have been observed $(2,3)$.

Genetic studies of patients with isolated hypoaldosteronism have frequently found mutations in the CYP11B2 gene, which encodes Aldo synthase (P450c11Aldo) (4-10). The patients with CMO II have occasionally been reported to have mutations that do not modify Aldo synthase activity $(2,11)$ or to have no mutations in coding regions at all $(2,12)$. On the contrary, the patients with CMO I have always been found to have some mutations that either completely inactivate the encoded CYP11B2 enzyme $(6,7,9,10)$ or significantly impair it (13).

The present case report represents an exception since it is, to our knowledge, the first CMO I case with no 
Table 1 Basal plasma hormone values of the patient and the reference range for her age.

\begin{tabular}{lcc}
\hline & Patient & Reference range \\
\hline Aldo $(\mathrm{pg} / \mathrm{ml})$ & 9.5 & $30-350$ \\
Plasma renin activity $(\mathrm{mg} / \mathrm{ml}$ per $\mathrm{h})$ & 27 & $2.2-10.2$ \\
Cortisol $(\mathrm{ng} / \mathrm{ml})$ & 191 & $130-260$ \\
18-OH-B $(\mathrm{pg} / \mathrm{ml})$ & 20 & $60-850$ \\
Corticosterone/18-OH-B ratio & 306.5 & $10-40$ \\
18-OH-B/aldo ratio & 2.4 & $5-10$ \\
\hline
\end{tabular}

mutations in the CYP11B2 gene, a molecular pattern which has been reported hitherto only in CMO II patients $(2,12)$.

\section{Case report}

This study concerns a girl born at 40 weeks of gestation from healthy and non-consanguineous parents. Both birth weight $(3.7 \mathrm{~kg})$ and length $(50 \mathrm{~cm})$ were normal. The first weeks of life were uneventful. At the age of 14 days she was admitted to another hospital, because of diarrhoea with severe salt-wasting syndrome (serum $\mathrm{Na}=125, \mathrm{~K}=8.6 \mathrm{mEq} / \mathrm{l})$. Although genitalia were normal and 17-hydroxyprogesterone (17-OH-P) levels were only slightly supranormal $(6.4 \mathrm{ng} / \mathrm{ml}$, normal range 0.03-1.55), diagnosis of 21-hydroxylase deficiency was assessed. The onset of both hydrocortisone and $9 \alpha$-fluorohydrocortisone therapy was followed by a rapid and persistent resolution of the salt-wasting clinical picture. Nevertheless, at the age of 60 days she was admitted (for the first time) to our clinic for a re-evaluation of diagnosis: no signs of virilization were observed and very low hydrocortisone doses $(1.25 \mathrm{mg} /$ day) were sufficient to maintain normal 17-OH-P serum levels. Both hydrocortisone and $9 \alpha$-fluorohydrocortisone were withdrawn for some days in order to investigate adrenal function.

An i.v. adrenocorticotrophin stimulation test elicited a normal 17-OH-P response (from baseline 0.5 to $1.0 \mathrm{ng} / \mathrm{ml}$ ). Moreover, Aldo serum levels were repeatedly low, ranging from 8.3 to $11.1 \mathrm{pg} / \mathrm{ml}$ (Table 1 ). On the basis of such biochemical results 21-hydroxylase deficiency was excluded and diagnosis of isolated hypoaldosteronism was definitely assessed.

According to the nomenclature proposed by Ulick (11), diagnosis of CMO I deficiency was more precisely established, in the light of the following criteria: very low 18-OH-B serum levels, an abnormally high corticosterone/18-OH-B serum ratio and an abnormally low 18-OH-B/Aldo serum ratio (Table 1 ).

After establishing the diagnosis of CMO I deficiency, hydrocortisone therapy was withdrawn whilst treatment with $9 \alpha$-fluorohydrocortisone was begun again, with a satisfactory clinical and metabolic impact. During the next 3 years no salt-wasting crisis was recorded and the patient's linear growth is persisting normally.

Direct sequences of the patient's DNA were able to identify only a heterozygous amino acid substitution in exon 7 of that gene: valine-386-alanine (V386A) GTG $\rightarrow$ GcG. No homozygous mutations within the exons and the exon/intron boundaries of the CYP11B2 gene have been observed.

\section{Methods}

\section{Steroid determinations}

Plasma steroids were measured using a method for the simultaneous determination of multiple adrenosteroids (by radioimmunoassay after extraction and automated Sephadex LH20 multicolumn chromatography) in a small volume of plasma (1-2 ml) (14).

\section{Nucleotide sequences of exons and exon/intron boundaries}

Genomic DNA was extracted from peripheral blood leucocytes and the CYP11B2 gene was specifically amplified by PCR in two fragments containing the 9 exons. Regions of CYP11B2 gene having extensive mismatches with CYP11B1 were used for the synthesis of primers. PCR products were treated before sequencing using exon-nuclease I and shrimp alkaline phosphatase. The nucleotide sequence of both strands of the PCR products was directly determined by thermocycle sequencing using the Thermo Sequenase radiolabelled terminator cycle sequencing kit following the manufacturer's instructions (Amersham Life Science, Cleveland, OH, USA). More details concerning the analysis of sequence can be found elsewhere ( 7 , 15).

\section{Discussion}

The present study seems to be inconsistent with the previously reported correlation of the phenotype and genotype in CMO type I.

Clinically our patient exhibited a severe salt-wasting syndrome during the first weeks of life, with no genitalia abnormalities: a pattern typical of both CMO I and CMO II deficiency. Biologically, we observed very low 18-OH-B serum levels associated with an increased corticosterone/18-OH-B ratio and a subnormal $18-\mathrm{OH}-$ $\mathrm{B} /$ Aldo ratio, i.e. a biochemical pattern pathognomonic of CMO I.

The sequencing of the CYP11B2 gene was able to identify only a heterozygous V386A mutation in exon 7. Some cases with no mutations in this gene have already been reported in CMO II $(2,12)$. On the contrary, ours is the first report on a patient with CMO type I who does not carry any homozygous mutation in the entire CYP11B2 alleles. 
The lack of homozygous gene mutation in this case is surprising. It cannot be excluded that a dominant negative effect may result from this heterozygous mutation; it is to be underlined, however, that even in individuals homozygous for the same mutation this change has been demonstrated to have no evident clinical effects without the presence of additional sequence changes (13). A double homozygosis R181W and V386A has been described in patients with CMO II $(4,5)$ and three simultaneous homozygous mutations in the CYP11B2 gene (V386A, R173K and E198D) have been reported in twins with a clinical phenotype typical of the type I deficiency (13). In vitro the V386A mutation is known to cause only a small reduction in 18-hydroxylase activity (4).

To sum up, it is unlikely that the isolated mutation found in the CYP11B2 alleles of our patient can be the only cause of her phenotype. The isolated (homozygous) changes in the CYP11B2 gene which have been reported hitherto in CMO I are: V35 del TGCTC (10), E255X (6, 15), R384P (7) and L461P (9). All these lesions are known to completely abrogate the activities of the encoded enzyme. Consequently Aldo is commonly undetectable in the serum of CMO I patients. In the present case report the presence of some measurable Aldo seems to suggest an intermediate form of the deficiency that might be compatible with the absence of homozygous mutations in the gene coding Aldo synthase. In our patient, the enzyme activity is likely not to be completely abolished, compared with that frequently observed in patients with both CMO I and documented mutations in the CYP11B2 gene. A residual activity of Aldo synthase may account for the low but detectable Aldo serum levels found in our patient.

The disparity between our patient's genotype and her biochemical phenotype typical of CMO type I, however, suggests that phenotype-genotype relationships in isolated hypoaldosteronism are not yet fully understood, as already postulated by other authors in the light of their findings in CMO II patients (13).

Our patient's disease could be due to other undetected mutations in the CYP11B2 gene, such as a mutation in the promoter or in an intron which disrupts splicing. It may be possible that a mutation is located in the $5^{\prime}$ flanking region of the CYP11B2 gene, which contains many regulatory elements. Alternatively, a defect in an adrenal-cell specific transcriptional factor that is necessary for the transcription of CYP11B2 could exist: upregulation of adrenal cell-specific repressors or a mutation in adrenal cell-specific repressors would allow for disease without a mutation in the coding region of CYP11B2 (16).

Finally, a reasonable question that might be raised on the basis of our findings in this case report is whether other genes, apart from CYP11B2, are involved in the regulation of terminal Aldo synthesis.

\section{Acknowledgements}

The authors are indebted to Professor W G Sippel (Kiel, Germany) for steroid determination and genetic analyses.

\section{References}

1 Ulick S. Diagnosis and nomenclature of the terminal portion of the aldosterone biosynthesis pathway. Journal of Clinical Endocrinology and Metabolism 197643 92-96.

2 Peter M, Nikischin W, Heinz-Erian P, Fussenegger W, Kapelari K \& Sippel WG. Homozygous deletion of arginine-173 in the CYP11B2 gene in a girl with congenital hypoaldosteronism (corticosterone methyl oxidase deficiency type II). Hormone Research $199850222-225$.

3 Peter M, Dubuis JM \& Sippell WG. Disorders of the aldosterone synthase and steroid 11 $\beta$-hydroxylase deficiencies. Hormone Research 199951 211-222.

4 Pascoe L, Curnow KM, Slutsker L, Rosler A \& White PC. Mutations in the human CYP11B2 (aldosterone synthase) gene causing corticosterone methyl oxidase II deficiency. PNAS 1992 89 4996-5000.

5 Mitsuuchi Y, Kawamoto T, Rosler A, Naiki Y, Miyahara K, Toda $\mathrm{K}$ et al. Congenitally defective aldosterone biosynthesis in human: the involvement of point mutations of the P-450c18 gene (CYP11B2) in CMO II deficient patients. Biochemical and Biophysical Research Communications 1992182 974-979.

6 Peter M, Fawaz L, Drop SLS, Visser HKA \& Sippel WG. Hereditary defect in the biosynthesis of aldosterone: aldosterone synthase deficiency 1964-1997. Journal of Clinical Endocrinology and Metabolism 199782 3525-3528.

7 Geley S, Johrer K, Peter M, Denner K, Bernhardt K, Sippell WG et al. Amino acid substitution R384P in aldosterone synthase causes CMO-I deficiency. Journal of Clinical Endocrinology and Metabolism $1995 \mathbf{8 0} 424-429$.

8 Zhang G, Rodriguez H, Fardella CE, Harris DA \& Miller WL. Mutation T318M in the CYP11B2 gene encoding P450c11AS (aldosterone synthase) causes corticosterone methyl oxidase II deficiency. American Journal of Human Genetics 199557 10371043.

9 Nomoto S, Massa G, Mitani F, Ishimura Y, Miyahara K, Toda K et al. CMO I deficiency caused by a point mutation in exon 8 of the human CYP11B2 gene encoding steroid 18-hydroxylase (P450c18). Biochemical and Biophysical Research Communications $1997234382-385$.

10 Mitsuuchi Y, Kawamoto T, Miyahara K, Ulick S, Morton HD, Naiki Y et al. Congenitally defective aldosterone biosynthesis in humans: inactivation of the P-450c18 gene (CYP11B2) due to nucleotide deletion in CMO I deficient patients. Biochemical and Biophysical Research Communications 1993190 864-869.

11 Ulick S. Correction of the nomenclature and mechanism of the aldosterone biosynthetic defects. Journal of Clinical Endocrinology and Metabolism $1996 \mathbf{8 1} 1299-1300$.

12 Fardella CE, Hum DW, Rodrigues H, Zhang G, Barry FL, Ilicki A et al. Gene conversion in the CYP11B2 gene encoding $\mathrm{P} 450 \mathrm{c} 11 \mathrm{AS}$ is associated with, but does not cause, the syndrome of corticosterone methyl oxidase II deficiency. Journal of Clinical Endocrinology and Metabolism $199681321-326$.

13 Portrat-Doyen S, Tourniaire J, Richard O, Mulatero P, AupetitFaisant B \& Curnow KM et al. Isolated aldosterone synthase deficiency caused by simultaneous E198D and V386A mutations in the CYP11B2 gene. Journal of Clinical Endocrinology and Metabolism $1998 \mathbf{8 3} 4156-4161$.

14 Sippel WG, Bidlingmaier F, Becker $H$, Bruning T, Dorr $H$, Hahn $\mathrm{H}$ et al. Simultaneous radioimmunoassay of plasma aldosterone, corticosterone, 11-deoxycorticosterone, progesterone, 17-hydroxyprogesterone, 11-deoxycortisol, cortisol and cortisone. Journal of Steroid Biochemistry 19789 63-74. 
15 Peter M, Bunger K, Drop SLS \& Sippell WG. Molecular genetic study in two patients with congenital hypoaldosteronism (types I and II) in relation to previously published hormonal studies. European Journal of Endocrinology 1998139 96-100.

16 Pilon C, Mulatero P, Barzon L, Veglio F, Garrone C, Boscaro M et al. Mutations in CYP11B1 gene converting 11 $\beta$-hydroxylase into an aldosterone-producing enzyme are not present in aldosterone- producing adenomas. Journal of Clinical Endocrinology and Metabolism 199984 4228-4231.

Received 17 February 2000

Accepted 21 September 2000 